\section{Painless computer-controlled experimentation}

A. B. DURELL

Ontario Institute for Studies in Education, Toronto, Ontario, Canada

More Es might employ computers to control psychological experiments if computer languages suitable for experimental control were easier to learn. In fact, course author languages intended for writing instructional sequences offer many facilities desirable for experimental control. A particular language, CAN-4, is examined for its suitability, and its use in a concept learning experiment is illustrated.

The case for computer control of psychological experiments has been ably stated by Miller, Bregman, and Norman (1965) and by Uttal (1968); computer-controlled experiments have been reported by White (1962), Green (1963), Johnson (1967), and others. While the usefulness of a computer for elimination of $E$ effects, handling complex decision rules, data collection, motivating Ss, and demanding a clear definition of experimental objectives are widely chronicled by many proponents, Johnson has raised the most pointed list of disadvantages of computer use in experimental work. His main objections are based on cost and the inflexibility of computers in handling human errors. The falling prices of computer hardware, rising wages of people (including Es), and the general applicability of computers to many tasks (Uttal, 1968) tend to answer the cost objection in a satisfactory manner. Of course, commercial time-sharing services could be used for many experiments, thus removing any need to purchase a computer (Huesmann, 1969).

A solution of the second difficulty, inflexibility, demands basic research advances in the processing of natural language by machines.

\section{COMPUTER LANGUAGES} AND EXPERIMENTS

One obstacle in the way of more widespread use of computers to control experiments is the necessity for Es to learn a computer language or to obtain the services of someone well versed in the use of such a language. Assembly-level languages are very powerful, but they are difficult to learn and often require a greater understanding of computer operation than Es have the desire or the time to acquire. More easily learned languages, such as FORTRAN, are often poorly suited for interactive use. Other languages, such as FOCAL, are designed for interactive applications but are not suitable for other purposes. One reason for this is that such languages are very difficult to modify or to trick into performing tasks beyond the scope of the applications for which they were originally intended. When modified, these languages are often inefficient in performing the new task.

Special languages, such as PSYCHOL (McLean, 1969), have been developed to control psychological experiments. Restle and Brown (1969) reported on a complete, computer-controlled laboratory which offers software support and a common experimental format to users. These approaches should become more common at large centers in the future, but the high cost of a computer-oriented laboratory or the lack of generality of a special-purpose language will make them inappropriate for many situations.

There is a class of languages which is easy to learn, designed for interactive use, and offers attractive features for experimental purposes. These are the course-authoring languages developed for writing computer-assisted instruction (CAI) sequences. Not all such languages offer equal ease of use nor the same range of facilities, but CAN-4 (Churchill, Naess, \& Olivier, 1971) combines simplicity with power very effectively.

\section{FACILITIES OF CAN-4}

In this section, some of the features of the language which offer potential for experimental applications will be discussed. The grouping is, in some cases, different from the logical

\section{Display}

CAN-4 allows the programmer to display textual and graphic material to Ss by means of a Teletype or CRT. A command also exists for controlling a slide projector, and another can access messages on a tape recorder. Thus, the language provides a number of ways of presenting stimuli to Ss which may be called upon for different experimental situations.

\section{Scoring Responses}

In common with the most rudimentary languages of its type, CAN-4 embodies routines which search for correct, incorrect, or unanticipated responses, so that arrangement of the language. keeping a score of the basic types of responses is very easy.
The available limited response processing features may be of value in providing an ability to process input strings, as long as the $E$ does not attempt to overextend what is still a very crude capability. As noted, any attempt at extensive processing of natural language is likely to be a futile pursuit with the present state of the art. The abilities of the CAN-4 language in this area seem as good as any, but they are still very limited. Their greatest value will likely lie in allowing some flexibility in the form of short responses, without having to write overly long sequences of possible responses for checking.

\section{Timing}

CAN-4 gives the $\mathrm{E}$ an ability to record response latencies, using the READ CLOCK command, or to limit the time available for the $S$ to respond. Recording response latencies is of such importance in many studies that its absence would probably render a language unsuitable for experimental applications.

\section{Compute}

Mathematical expressions in the program can be evaluated, using the value of variables at run time. Such a facility is of importance in running many types of contingent experiments. It also has value in allowing some simple statistics to be calculated during the run which determine if more trials are necessary. A pseudorandom number generator is also available.

\section{Subroutines}

Often experimental treatments will differ only in part or in the order in which segments of the method are applied. The ability to construct sections of the controlling program as subroutines makes it easy to combine portions of treatments in various arrangements and to avoid the duplication of extensive sections of code. This is also of importance when follow-up experiments are designed. Entire subroutines may be employed with little or no change in the code.

\section{Performance Files}

In common with most CAI languages, CAN-4 is designed to allow easy compilation of records of $S$ performance. In fact, every response made by a $S$, together with appropriate time measures, may be recorded automatically. 


\section{ADVANTAGES OF A}

COMPLETE SYSTEM

It is unlikely that any single experiment would use all of the features which CAN-4 offers. In this sense, there is some inefficiency in using such a language. However, this inefficiency seems to be more than counterbalanced by the simplicity of learning to write a program in CAN-4, the speed with which such a program can be written and modified, and the fact that the language makes available a tested and coordinated system. Very often in constructing an Assembly language program for a particular application, one finds that a subroutine has been written previously to perform one of the subtasks of the application. Unfortunately, it is common to find that the existing subroutine was written for a slightly different use, and the work of adapting it may be at least as great as that needed to write a new one. If more than one existing subroutine is incorporated into a program, such problems are likely to be multiplied.

Some of the greatest problems in constructing a program are those of $\mathrm{I} / \mathrm{O}$ and of opening and closing disk files. These problems are of little or no intrinsic interest to most Es. With a complete system, such as CAN-4 provides, these problems are handled automatically and the $\mathrm{E}$ can forget about them.

CAI languages provide the further advantage of being more generally available than complete laboratory systems such as that of Restle and Brown (1969) or the special-purpose language of McLean (1969). A representative group of papers dealing with CAI (Atkinson \& Wilson, 1969) provides many references to specific implementations of CAI systems. A comparison of many interactive languages used for instruction is given by Zinn (1969). This guide to the instructional value of languages may be of use in selecting languages of potential value for experimental control.

CAN-4 runs on a time-shared PDP-9 with $16 \mathrm{~K}$ core memory and two-million-word, fixed-head disks (Ensor, 1969; McLean, 1970; Ragsdale, 1970). A version of $\mathrm{CAN}-4$, written mainly in FORTRAN, is running on a time-shared PDP-10. AN EXAMPLE

The author conducted a study of the use of strategies in learning concepts (Durell, 1971). A CAN-4 program controlled instruction of Ss, presentation of the task, and the recording of data.

The task was adapted from an example of Scandura's (1968). Each S was presented with a triple of digits and a result in the form $(3,1,4): 4$, with the triple of digits contained in parentheses and the result following the colon. He knew that the result was produced from exactly two of the digits by addition, subtraction, or multiplication. The digits were selected on the basis of their position within the triple (first, second, or third), on the basis of relative magnitude (smallest, middle-sized, largest), or on a combination of the two. Thus, in the example above, some of the rules by which the result, 4 , might be produced from the triple of digits, $(3,1,4)$, are: first digit added to the second digit; smallest digit multiplied by the largest digit; first digit added to the smallest digit.

To determine which concept (rule) is exemplified, the $\mathbf{S}$ constructed his own triples and results. The program examined them to determine if they were exemplars of the concept and informed the $S$.

When the $S$ felt that he knew the concept, he requested a test. The test consisted of prepared triples of digits which were presented. The S supplied the result, using the concept he had in mind. If the $s$ provided five consecutive correct results, he was assumed to know the concept and was advanced to the next concept to be learned.-If he made an error, he was branched to further trials until he again requested the test.

The use of branching and subroutines allowed one overall routine to control the presentation of concepts, as well as the reception and recording of responses. Individual subroutines for each concept examined the instances proposed by the $\mathbf{S}$ to determine whether they were positive or negative instances of the concept.

At the same time, measures of response latencies were recorded in order that the S's strategy and tempo of responding might be examined for relationships.

The variables provided by the language were used to record number of responses, latencies, and errors on test items, as well as serving as pointers to subroutines and branches, depending on the concept being considered.

\section{CONCLUSION}

For some experiments, computer control can offer substantial advantages. For experiments which can be accommodated by the time-sharing mode, CAI languages may provide a vehicle by which many more Es can begin this type of work. In particular, CAN-4 seems to offer an array of facilities which are well suited to experimental control applications.

\section{REFERENCES}

ATKINSON, R. C., \& WILSON, H. A Computer-assisted instruction: $A$ book of readings. New York: Academic Press, 1969.

CHURCHILL, S., NAESS, L \& OLIVIER, W. P. CAN-4, an advanced author language for $\mathrm{CAI}$, computer-based testing and psychological experimentation: PDP-9 implementation. Behavior Research Methods \& Instrumentation, 1971, 3, 95-99.

DURELL, A B Teaching concept acquisition strategies for mathematics. Unpublished MA thesis, University of Toronto, 1971.

ENSOR, D. A. A time-sharing system for the PDP-9. Paper given at DECUS 1969 fall symposium.

GREEN, B. F., JR. Digital computers in research: An introduction for behavior and social scientists New York: McGraw-Hill, 1963.

HUESMANN, L. R. Controlling experiments with a general-purpose time-sharing system. Behavior Research Methods \& Instrumentation, 1969, 1, 276-280.

JOHNSON, E. S. The computer as experimenter. Behavioral Science, 1967. 12, 484-489.

McLEAN, R. S. PSYCHOL: A computer language for experimentation. Behavior Research Methods \& Instrumentation, 1969, 1, 323-328.

McLEAN, R. S. TSS-9 revisited. Paper given at DECUS 1970 spring symposium.

MILLER, G. A., BREGMAN, A. S., \& NORMAN, D. A. The computer as a general purpose device for the control of psychological experiments. In R. W. Stacy and B. Waxman (Ed.), Computers in biomedical research. Vol. I. New York: Academic Press, 1965. Pp. 467-490.

RAGSDALE, R. G. TSS-9 status report. Paper given at Canadian DECUS sy mposium, 1970.

RESTLE, F.. \& BROWN, T. V. A computer running several psychological laboratories. Behavior Research Methods \& Instrumentation, 1969, 1, 312-317.

SCANDURA, J. M. Research in psychomathematics. Mathematics Teacher, 1968, 56, 581-591.

U T T A L, W. R. Real-time computers-Technique and applications in the psychological sciences. New York: Harper \& Row, 1968.

WHITE, B. J. Studies of perception. In H. Borko (Ed.), Computer applications in the behavioral sciences. Englewood Cliffs. N.J: Prentice-Hall, 1962. Pp. 280-307.

ZINN, K. L. Comparative study of languages for programming interactive use of computers in instruction. Boston: EDUCOM, February 1969. 